\title{
Control of the flow in the annular region of a shrouded cylinder with splitter plate
}

\author{
Gokturk Memduh Ozkan ${ }^{1,}$, Tahir Durhasan ${ }^{1}$, Engin Pinar $^{2}$, Arda Yenicun ${ }^{1}$, Huseyin Akilli ${ }^{1}$ and Besir Sahin ${ }^{1}$ \\ ${ }^{1}$ Faculty of Engineering and Architecture, Department of Mechanical Engineering, Çukurova University, 01330, Adana, Turkey \\ ${ }^{2}$ Faculty of Ceyhan Engineering, Department of Mechanical Engineering, Çukurova University, Ceyhan, Adana, Turkey
}

\begin{abstract}
In the present study, the flow control with a splitter plate was studied considering the annular region of a shrouded cylinder. The effect of splitter plate angle, $\alpha$ which was defined according to the cylinder centreline is investigated experimentally in deep water using Particle image Velocimetry (PIV) technique and flow visualization by dye injection method. The range of splitter plate angle was selected within $60^{\circ} \leq \alpha \leq 180^{\circ}$ with an increment of $30^{\circ}$. The porosity of the shroud which is a perforated cylinder was selected as $\beta=0.7$ in order to have larger fluid entrainment through the cylinder. The results were compared with the no-plate case and showed that the splitter plate located in the annular region of shrouded cylinders is effective on reducing the turbulence levels just behind the cylinder base, as well as the near wake of the perforated shroud.
\end{abstract}

\section{Introduction}

The flow around bluff bodies has been extensively studied in order to analyse the effects of unsteady flow on the integrity of even large engineering structures. The cylinder as a bluff body has been preferred by many researchers where the vortex shedding from the cylinder causes fluctuations in pressure forces. According to this condition, vibration, noise or resonance are produced on the cylinder which should be controlled. The aims of controlling the flow around cylinder are to reduce drag, magnitude of the fluctuating force and time-varying flowinduced forces acting on bluff bodies. Flow control is normally classified into active and passive control in which the active methods require energy input whereas the passive methods modify the flow structure of interest and no energy input required. Therefore the passive control techniques are more preferred in practical applications. There is an abundance of studies in the literature on the flow past a circular cylinder starting with Karman [1] and Strouhal [2]. Roshko [3] used splitter plate placed behind the cylinder to control the flow structure. Price [4] used different shaped shrouds in order to control vortex induced vibration in his thesis which is about the effect of different geometries on flow control. Every et al. [5] reviewed the practical aspects of vortex excited structural vibrations in sub-sea water applications with particular emphasis on methods of their suppression. In a part of their study, they focused on the effect of shrouds on the flow control, reported a comprehensive literature and stated that shrouds with the optimum dimension gave a $50 \%$ reduction in oscillations of a plain study on suppressing flow induced vibrations.
A similar logic is being used in recent years called porous media which includes the idea of generating a permeable medium around a bluff body. An emerging approach of flow control is to attach a porous layer to the external surface of a structure. Bruneau and Mortazavi [6, 7] investigated the flow control by using porous layer around a cylinder numerically. They used penalization method and found that adding a porous ring around a riser pipe can damp the vortex induced vibrations. The study of Ozkan et al. [9] presents an example of flow control by shrouded cylinder in shallow water. They investigated the flow around a cylinder (inner cylinder) by a permeable outer cylinder having different porosities, $\beta$ and diameter ratios, $D / d$. They stated that the permeable outer cylinder suppresses the organized vortex street by reducing the velocity fluctuations in the near wake of the cylinder. The flow structure around perforated circular cylinders was studied experimentally by Pinar et al. [10]. Their study has shown that the porosity, $\beta$ has a significant impact on the unsteady flow structure downstream of the cylinder. The jet-alike flow through the holes on the surface of the perforated cylinders effectively prevents the formation of a well-organized Karman vortex street. With increasing porosity, the vortices formed on the upper and lower shear layers are elongated along the flow direction by losing their magnitudes compared to the plain cylinder case. Recently, Durhasan et al. [11] controlled the flow around a circular cylinder using semi-circular cylinders which are concentrically located. They performed their experiments considering various porosity values and arc angles and found that the perforated semi-circular fairings are effective on reducing turbulent statistics. Molin [8] performed a numerical study about perforated 
shrouds used to reduce vortex-induced vibrations of cylinders. He concluded that the proposed theory cannot be said to predict reliable values of the drag of shrouded cylinders since it fails to reproduce correctly the flow in the downstream part of the annular region, in-between the shroud and cylinder.

Within the light of Molin's [8] conclusion and the results of Ozkan et al. [12] the focus on the annular region of shrouded cylinder is taken into consideration for the current study. A splitter plate is located in the annular region of perforated shroud with varying angle to prevent the interaction of vortices generated by the inner cylinder.

\section{Experimental Method}

Both PIV and dye visualization experiments were performed in a large-scale closed-loop recirculating water channel, located in the Mechanical Engineering Department of Çukurova University having a test section of $1 \mathrm{~m}$ width $\times 0.75 \mathrm{~m}$ height and $8 \mathrm{~m}$ length made of a $15 \mathrm{~mm}$ thick transparent Plexiglas sheet. Free stream velocity was controlled by a $15 \mathrm{~kW}$ centrifugal pump with speed control unit which was used to adjust pump frequency. Honeycomb arrangements were located inside the channel in order to minimize the free-stream turbulence, which is expected to be less than $1 \%$. The free stream velocity was taken as $100 \mathrm{~mm} / \mathrm{s}$ which corresponds to a Reynolds number of $\mathrm{Re}_{\mathrm{d}}=5000$ according to the cylinder diameter. All experiments were performed above a platform, having a length of $2.3 \mathrm{~m}$. Perforated shroud is placed at the distance of $1.8 \mathrm{~m}$ from the leading edge of the platform to provide fullydeveloped boundary layer flow. During all experiments, the total depth of the water in the channel was kept constant as $0.6 \mathrm{~m}$. A schematic view of the experimental setup is shown Fig.1.

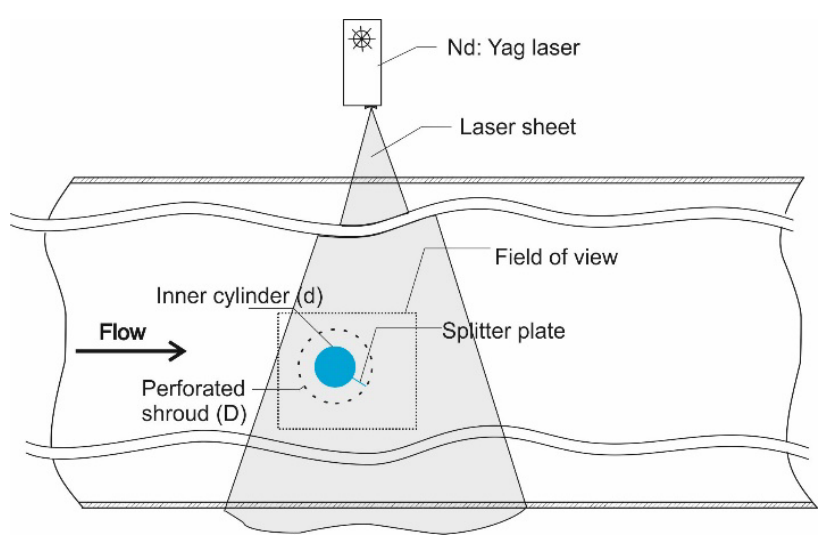

Fig. 1. Plan view of the experimental set-up

The geometric blockage of the perforated shroud was $10 \%$ with respect to the width of test section. Chromenickel metals sheets with $1 \mathrm{~mm}$ thicknesses were drilled using laser cutting machine to have $\mathrm{d}=10 \mathrm{~mm}$ holes on the cylinder surface, then they were rolled to manufacture the perforated shroud. The number of the holes on the shroud is expected to have significant effects on the flow structure. Therefore, the porosity ratio, $\beta$ defined as the ratio of the total open area on the cylinder to the whole cylinder surface area was chosen as a dimensionless parameter. Fig. 2 shows the schematic representation of the model and calculation of the porosity, $\beta$. Here the inner cylinder, splitter plate and perforated shroud dimensions are $\mathrm{d}=0.05 \mathrm{~m}, \mathrm{~L}=0.05 \mathrm{~m}$ and $\mathrm{D}=0.1 \mathrm{~m}$, respectively. The porosity, $\beta$ value was taken constant as $\beta=0.7$ through the experiments.

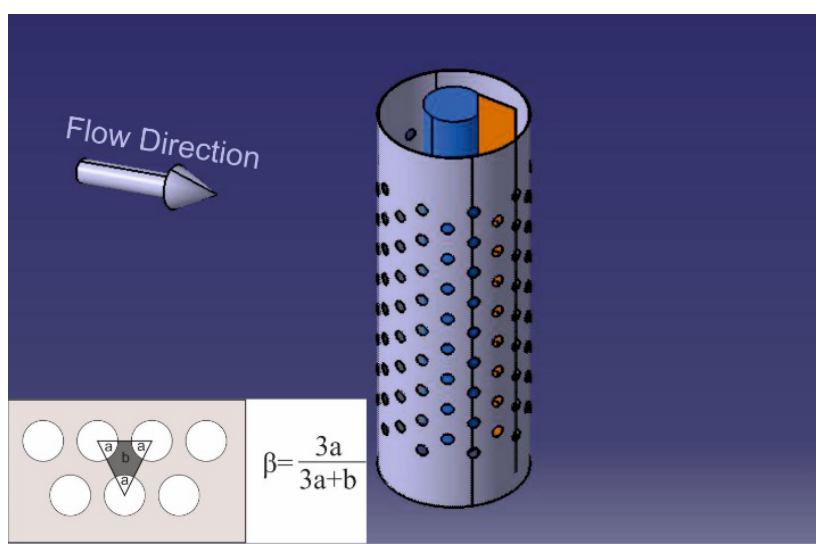

Fig. 2. Schematic representation for the model and the definition of porosity, $\beta$

The laser sheet was adjusted at the half of the water level and parallel to the bottom wall of the channel. Experiments were conducted in two steps: In the first step, flow visualization experiments were performed using Rhodamine type dye that shines under the continuous laser beam in the desired flow field. Throughout the experiments, dye was supplied at a location where the flow was not affected by the injection. Visualization of the experimental results was recorded with a high speed digital video recorder (SONY 80X handycam) to examine the flow characteristics in detail. Next, the Particle Image Velocimetry (PIV) technique was used in order to measure the velocity vector fields. Experiments were focused on the region between the cylinder and perforated shroud (which will be called as annular region). The measurement field was illuminated by a thin and intense laser light sheet using double-pulsed Nd:YAG laser units. Each pulse has a nominal energy output of $120 \mathrm{~mJ}$ at $532 \mathrm{~nm}$ wave length. The time interval between pulses was $1.75 \mathrm{~ms}$ for all experiments and the thickness of the laser sheet illuminating the measurement plane was approximately $2 \mathrm{~mm}$. The time interval and the laser sheet thickness were selected such that the maximum amount of particle displacement in the interrogation window was obtained. The water was seeded with $10 \mu \mathrm{m}$-diameter seeding particles and the particle density was about $1100 \mathrm{~kg} / \mathrm{m}^{3}$. The number of particles in an interrogation area was in between 15 and 20. The experimental measurements were performed and the data were processed using Dantec Dynamics PIV system and Dynamics Studio Software installed on a 
computer. In each experiment, 1000 instantaneous images were captured at a rate of $15 \mathrm{~Hz}$, recorded and stored in order to obtain time-averaged velocity vectors. Assuming isotropic flow, the values of turbulent kinetic energy (TKE) were evaluated using the estimation of Sheng et al. [13] which states that the third component has been supposed to be equal to the half of $\left(\left\langle u^{\prime} u^{\prime}\right\rangle+\left\langle v^{\prime} v^{\prime}\right\rangle\right)$ and TKE can be calculated using $<T K E>=3 / 4\left(<u^{\prime} u^{\prime}>+<v^{\prime} v^{\prime}>\right)$. The image capturing was performed by an 8-bit cross-correlation CCD camera having a resolution of $1600 \times 1200$ pixels, equipped with a Nikon AF Micro $60 \mathrm{f} / 2.8 \mathrm{D}$ lens. In the image processing, $32 \times 32$ rectangular interrogation pixels were used and an overlap of $50 \%$ was employed. Shadows, reflections or laser sheet distortions etc. cause spurious velocity vectors in the flow field, therefore a local median-filter technique was employed to erase spurious vectors (less than $3 \%$ ) in which they were replaced using bilinear interpolation between neighbouring velocity vectors. The velocity vector field was also smoothed to avoid dramatic changes in the velocity field using the Gaussian smoothing technique. To minimize distortion of the velocity field, a smoothing parameter of 1.3 was chosen. The details of the effects of these factors can be found in the studies of Fouras and Soria [14], Adrian [15] and Keane and Adrian [16]. Finally, the instantaneous and mean vorticity maps (vorticity value at each grid point was calculated from the circulation around eight neighbouring point) and turbulence statistics were determined as a result of postprocessing operation.

\section{Results and Discussion}

The pictures evaluated from dye visualization experiments are presented in Fig. 3 where the flow behaviour is clarified by the red lines showing the vortical flow structures. Here the dashed outer line presents the perforated shroud located concentrically around the inner cylinder. In order to interpret the difference, the results of shrouded cylinder without any splitter plate (no-plate case) are also presented on the first picture. An incoherent vortex pair is observed within the annular region downstream of the inner cylinder which might increase the turbulence. As a result, even the vortex shedding is controlled downstream of the shrouded cylinder, there would be vortex shedding in the near wake of inner cylinder. To get rid of this undesirable effect, a splitter plate is located in between inner cylinder and the shroud and presented by letter pictures in Fig. 3 for various plate angles.
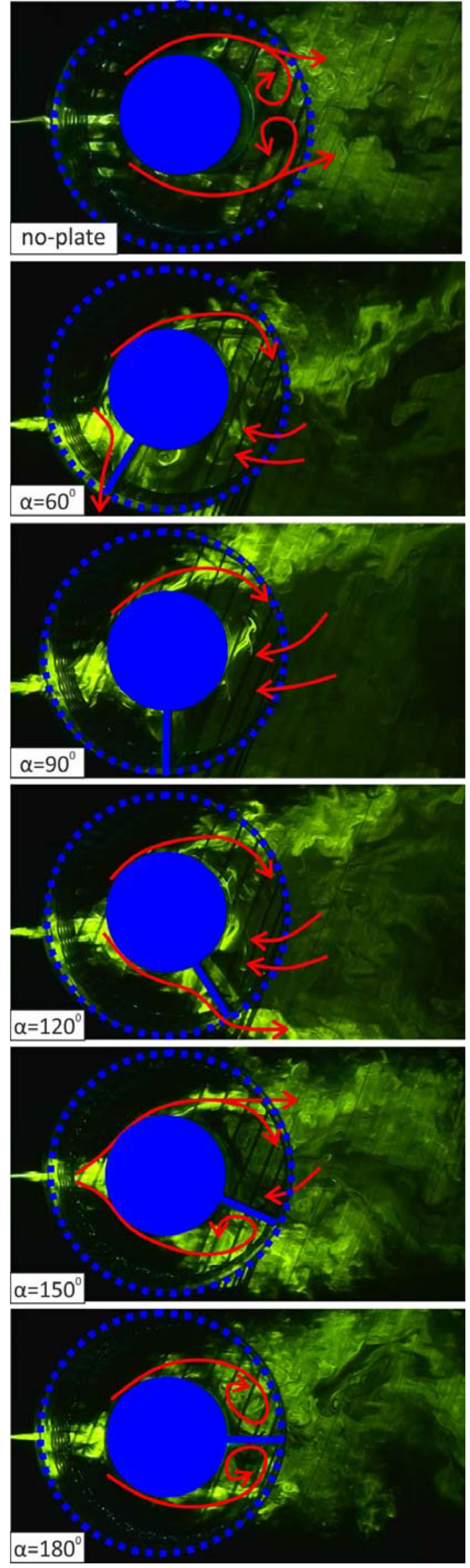

Fig. 3. Instantaneous pictures of the dye visualization experiments 
In Fig. 2, for the splitter plate angles of $60^{\circ} \leq \alpha \leq 120^{\circ}$, the incoming flow through perforated shroud is blocked by the plate where some amount of fluid leaves from the perforated shroud along the lower shear layer. Besides, a huge amount of fluid, hence momentum is transferred to the other side, i.e. upper shear layer of the inner cylinder. Since the porosity of the perforated shroud has a high value, most of the fluid emanate through the holes on the upper side and then recirculates in the wake of perforated cylinder, finally re-enters into the annular region as can be seen by $60^{\circ} \leq \alpha \leq 120^{\circ}$. Increasing the splitter plate angle to $\alpha=150^{\circ}$ brings out slight changes in flow structure, i.e. a recirculating flow exists in the lower side of the annular region which breaks through the holes. The angle value of $\alpha=180^{\circ}$ presents the well-known splitter plate method within the literature and might be the best way of preventing the interaction of vortices formed by the inner cylinder within the annular region. Here the vortices are more distinct compared with the no-plate case and their interaction is eliminated. Furthermore, the generated vortices are decomposed by the holes on the perforated shroud and cannot be seen in the near wake of the shroud.

The qualitative dye visualization results were analysed to understand the general behaviour of the flow which actually cannot be determinant for the accuracy of the method. Therefore it is compulsory to analyse the quantitative PIV results. Fig. 4 includes the timeaveraged streamlines superimposed with the vorticity contours for all cases. In consistent with the flow visualization pictures, a clear recirculation occurs within the annular region of shrouded cylinder for no-plate case. The focal points generated here are designated by $F_{1}$ and $\mathrm{F}_{2}$ within annular region whereas another focal point, $\mathrm{F}_{3}$ is generated in the downstream of perforated shroud. According to this result one can conclude that there exist vortex shedding within the annular region of the shrouded cylinder, as well as downstream of the perforated shroud. Therefore, use of a shroud with high porosity cannot be an accurate flow control technique. Because in this case both the inner cylinder and the cylinder-shroud configuration is under the effect of vortex shedding. To prevent this, locating a splitter plate may bring out a solution for the problem. Use of the splitter plate having a plate angle of $\alpha=60^{\circ}$ makes the two focal points away from each other, i.e. one is formed on the upper side close to the perforated shroud and the other is formed on the lower side close to the splitter plate. The results of $\alpha=90^{\circ}$ presents the best case where no recirculation exists for this plate angle, however the vorticity magnitude on the lower side of perforated shroud contacting with the leading edge of the plate dramatically increases due to the jet flow through the holes in that region. This is also valid for the case of $\alpha=60^{\circ}$ with lower vorticity magnitudes. The results of $\alpha=120^{\circ}$ similar with the lower plate angles except the formation of high magnitude vorticity layer on the leading edge of the plate. It should be pointed out that for $60^{\circ} \leq \alpha \leq 120^{\circ}$ the wake of the perforated shroud is highly effected by the use of splitter plate in the annular region, i.e. the width and length of the recirculation region is increased compared with the no-plate case.

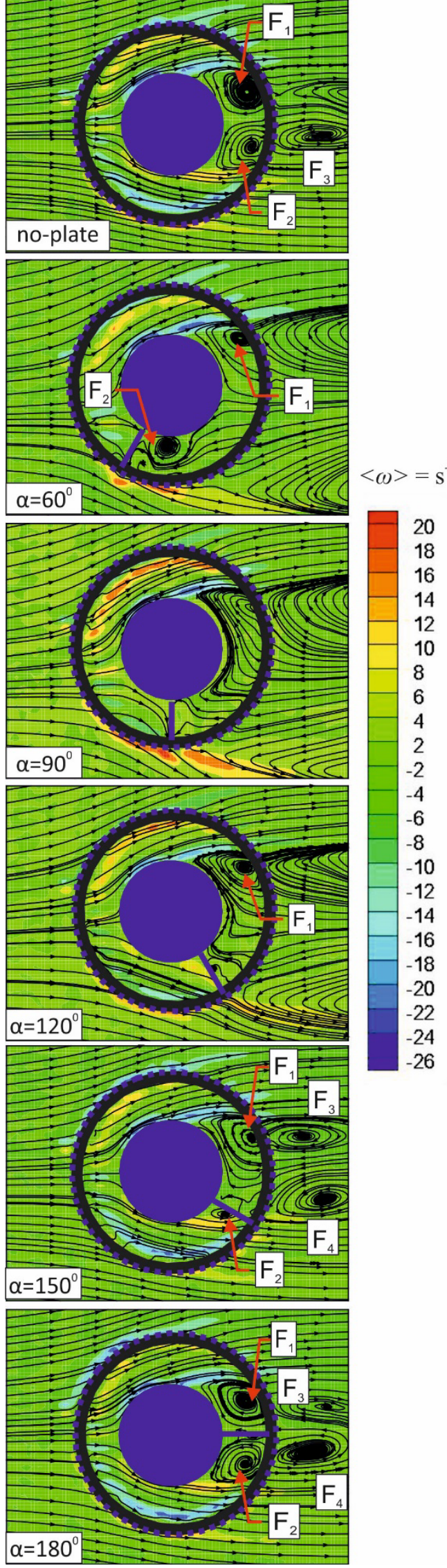

Fig. 4. Time-averaged streamlines superimposed with the vorticity contours for all cases 
For $\alpha>120^{\circ}$, similar flow structures with that of the no-plate case are obtained where two focal points are generated within the annular region and another pair of focal points are generated in the wake of perforated shroud. The use of a splitter plate with $\alpha=180^{\circ}$ completely prevents the interaction of vortex pairs generated by the cylinder, however there still exists the vortex pairs in the wake of perforated shroud which should also be eliminated.

In order to interpret the effectiveness of splitter plate on flow control within annular region, the focus on quantitative results passed to turbulence statistics by Fig. 5 . Here the time-averaged dimensionless turbulent kinetic energy, $<$ TKE $>$ concentrations are presented for the whole flow field where the minimum and incremental values for the turbulent kinetic energy were both taken as 0.005 . The aim of the study was to suppress the vortex shedding downstream of the inner cylinder within the annular region. One can easily see that the $<$ TKE $>$ values at upstream of the perforated shroud presenting the effect of incoming are highly concentrated for all cases. For the no plate case, the $<\mathrm{TKE}>$ concentrations are formed on upper and lower shear layer of the inner cylinder within the annular region. Furthermore, there exists intensive concentrations in the wake of perforated cylinder which also need to be diminished. When looking at the controlled cases with splitter plate, it can be seen that the lowest $<$ TKE $>$ values are achieved for the plate angle of $\alpha=120^{\circ}$, for both the annular region and the near wake of the perforated shroud. For larger plate angles, the TKE concentrations within the annular region are located in the very near wake of inner cylinder, however the upper and lower shear layer concentrations are separated from each other by the existence of splitter plate. The unfavourable condition for $\alpha=150^{\circ}$ and $\alpha=180^{\circ}$ is that the turbulence levels in the wake of the perforated shroud is still high as evaluated by the no-plate case. Therefore even the use of a splitter plate is preferable within the annular region, the flow generated in the wake of perforated shroud should also be controlled. It can be concluded by the TKE results that the best plate angle to reduce turbulence in the annular region downstream of the inner cylinder is $\alpha=120^{\circ}$ where the turbulence level is decreased remarkably compared with the no-plate case.

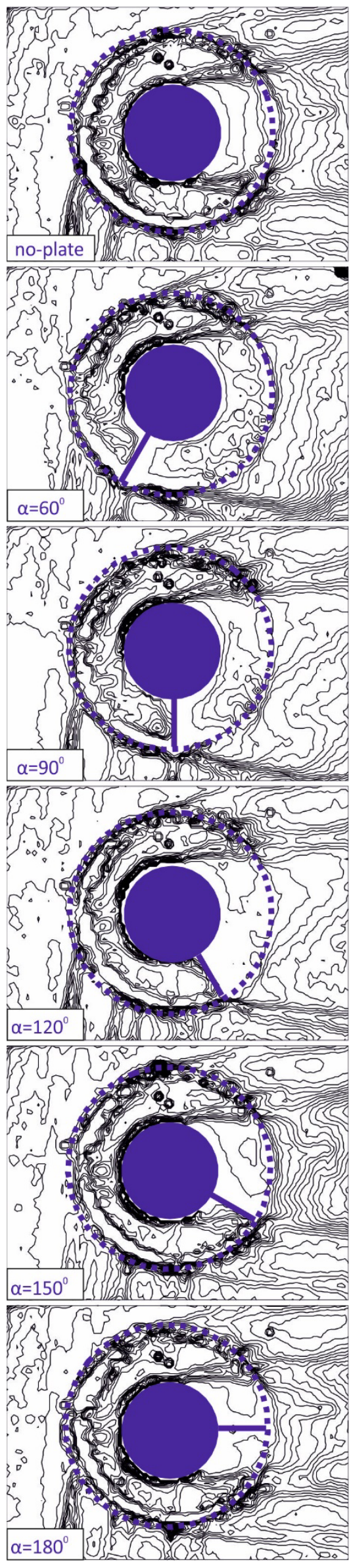

Fig. 5. Time averaged turbulent kinetic energy, $<\mathrm{TKE}>$ distributions for all cases 


\section{Conclusions}

The flow within the annular region of a shrouded cylinder was studied and the control of the unsteady vortical flow generated by the inner cylinder was analysed within this work. A splitter plate was located in the annular region and the angle of the plate was varied with respect to the cylinder centreline. The effect of the splitter plate angle on the flow structure within annular region, as well as the near wake of the perforated shroud were analysed using flow visualization and PIV techniques.

The results revealed that the use of a splitter plate with the plate angles of $60^{\circ} \leq \alpha \leq 120^{\circ}$ increases the wake width in the near wake of the perforated shroud by decreasing the turbulence levels in the annular region. The best reduction in the turbulence was evaluated for the plate angle of $\alpha=120^{\circ}$ where the turbulence concentrations are lower in the near wake of the cylinder, as well as the perforated shroud. Therefore the splitter plate at an angle of $\alpha=120^{\circ}$ could be advised for the current method.

Furthermore, the cases of $\alpha=150^{\circ}$ and $\alpha=180^{\circ}$ also be advised within the aid of a complete elimination of upper and lower vorticity layer interactions in the annular region. However for these cases, the turbulence levels were found to be still high in the near wake of the perforated shroud which remains as a problem to be solved or controlled by the future works.

\section{Acknowledgements}

The funding of the Scientific and Technological Research Council of Turkey under contract number $114 \mathrm{R} 087$ is greatly appreciated.

\section{References}

1. T. Karman, Matematisch-Physikalische Klasse, Göttingen Nachrichten, Über der Mechanismus des Wiederstandes, den eine bewegter Körper in einer Flüssigkeit erfahrt. 547-556 (1912)

2. V. Strouhal. Annelen für Physik und Chemie, Über eine besondere Art der Tonerregung, (On an unusual sort of sound excitation), 241, 216-251 (1878).

3. A. Roshko, Journal of Wind Engineering and Industrial Aerodynamics, Perspectives on bluff body aerodynamics. 49, 79-100 (1993).

4. P. Price, J. Eng. Mech. Div., Am. Soc. Civ. Eng, Suppression of the fluid-induced vibration of circular cylinders, 82, 1030 (1956).

5. M. J. Every, R. King, D. S. Weaver, Ocean Engineering, Vortex-Excited vibration of cylinder and cables and their suppression, 9, 135-157 (1982).

6. C-H Bruneau, I. Mortazavi, International Journal for Numerical Methods in Fluids, Passive control of the flow around a square cylinder using porous media, 45, 415-433 (2004).

7. C-H Bruneau, I. Mortazavi, Computers and Fluids, Numerical modelling and passive flow control using porous media, 37, 488-498 (2008).

8. B. Molin, Journal of Fluids and Structures, A potential flow model for the drag of shrouded cylinders, 7, 2938 (1993).

9. G.M. Ozkan, V. Oruc, H.Akilli, B. Sahin, Experiments in Fluids, Flow around a cylinder surrounded by a permeable cylinder in shallow water, 53, 1751-1763 (2012).

10. E. Pinar, G.M. Ozkan, T. Durhasan, M.M. Aksoy, H. Akilli, B. Sahin, Journal of Fluids and Structure, Flow structure around perforated cylinders in shallow water, 55, 52-63 (2015).

11. T. Durhasan, M.M. Aksoy, E. Pinar, G.M. Ozkan, H. Akilli, B. Sahin, Vortex street suppression of a circular cylinder using perforated semi-circular fairing in shallow water Experimental Thermal and Fluid Science, 79, 101-110 (2016).

12. G.M. Ozkan, H.Akilli, B. Sahin, EPJ Web of Conferences, Effect of High Porosity Screen on the Near Wake of a Circular Cylinder, 45, 01071 (2013).

13. J.Sheng, H. Meng, R.O. Fox, Chemical Engineering Science, A large eddy PIV method for turbulence dissipation rate estimation, 55, 4423-4434 (2000)

14. A. Fouras, J. Soria, Exp. Fluids, Accuracy of out-ofplane vorticity measurements derived from in-plane velocity field data, 25, 409-430 (1998).

15. R.J. Adrian, Annul. Rev. Fluid Mech., Particleimaging techniques for experimental fluid mechanics 23, 261-304 (1991).

16. R.D. Keane, R.J. Adrian Opt. Methods Flow Particle Diagnostics, Optimization of particle image velocimeters, 68, 139-159 (1989). 